\title{
Transient relay function of midline thalamic nuclei during long-term memory consolidation in humans
}

\author{
Jan-Willem Thielen, ${ }^{1,2,3}$ Atsuko Takashima, ${ }^{1,4}$ Femke Rutters, ${ }^{5}$ Indira Tendolkar, ${ }^{1,2,6}$ \\ and Guillén Fernández ${ }^{1,7}$ \\ ${ }^{1}$ Donders Institute for Brain Cognition and Behavior, Radboud University Medical Center, 6537 Nijmegen, The Netherlands; \\ ${ }^{2}$ Department for Psychiatry and Psychotherapy, Faculty of Medicine, University of Duisburg-Essen, 45127 Essen, Germany; ${ }^{3}$ Erwin \\ L. Hahn Institute for Magnetic Resonance Imaging, 45127 Essen, Germany; ${ }^{4}$ Behavioural Science Institute, Radboud University, \\ 6537 Nijmegen, The Netherlands; ${ }^{5}$ Department of Epidemiology and Biostatistics, VU University Medical Center, 1081 Amsterdam, \\ The Netherlands; ${ }^{6}$ Department of Psychiatry, Radboud University Medical Center, 6537 Nijmegen, The Netherlands; 7 Department of \\ Cognitive Neuroscience, Radboud University Medical Center, 6537 Nijmegen, The Netherlands
}

To test the hypothesis that thalamic midline nuclei play a transient role in memory consolidation, we reanalyzed a prospective functional MRI study, contrasting recent and progressively more remote memory retrieval. We revealed a transient thalamic connectivity increase with the hippocampus, the medial prefrontal cortex (mPFC), and a parahippocampal area, which decreased with time. In turn, mPFC-parahippocampal connectivity increased progressively. These findings support a model in which thalamic midline nuclei serve as a hub linking hippocampus, mPFC, and posterior representational areas during memory retrieval at an early $(2 \mathrm{~h})$ stage of consolidation, extending classical systems consolidation models by attributing a transient role to midline thalamic nuclei.

\begin{abstract}
The standard model of systems consolidation (e.g., Alvarez and Squire 1994) describes an initial stage in which the hippocampus binds together distributed neocortical representations during retrieval of recent memories. These neocortical representations code event features that are located in sensory-specific and multimodal representational areas in posterior parts of the brain, which are engaged during initial encoding. With consolidation, interconnectivity between these representational areas stabilizes, allowing retrieval of remote memories to be hippocampally independent. Accumulating evidence from animal and human work has extended this view by showing evidence for medial prefrontal cortex (mPFC) involvement during remote memory retrieval (Bontempi et al. 1999; Takashima et al. 2006; TakeharaNishiuchi and McNaughton 2008). The mPFC appears interacting with posterior representational areas during retrieval of remote memories (Frankland and Bontempi 2005), potentially binding them together (Takashima et al. 2006; Wheeler et al. 2013).

The systems-level processes underlying this transition from a hippocampally to a neocortically dependent memory are not well understood, though there seems evidence for a hippocampal$\mathrm{mPFC}$ interaction underlying this process (van Kesteren et al. 2010). However, rodent hippocampal-mPFC fibers are unidirectional and rather unevenly distributed over the hippocampus (Vertes et al. 2007; Hoover and Vertes 2012) and thus, an indirect interaction appears necessary if a bidirectional information flow between $\mathrm{mPFC}$ and hippocampus is required during systems consolidation. The rat's thalamic midline structures (nucleus reuniens/rhomboid nucleus) are reciprocally connected to both the $\mathrm{mPFC}$ and hippocampus as well as to posterior representational areas (Aggleton and Brown 1999; Van der Werf et al. 2002; Vertes et al. 2006, 2007; Hoover and Vertes 2012; Cassel et al. 2013; Wheeler et al. 2013) that seems also evident in nonhuman
\end{abstract}

Corresponding author: jan-willem.thielen@uni-due.de Article is online at http://www.learnmem.org/cgi/doi/10.1101//m.038372. 115. primates (Amaral and Cowan 1980; DeVito 1980; Aggleton et al. 1986, 2011; Hsu and Price 2007). Indeed, the nucleus reuniens acts as a hippocampal-mPFC relay during memory encoding in mice determining specificity/generalization of memory attributes (Xu and Südhof 2013). The nucleus reuniens seems also to be important in offline consolidation processes that occur immediately after memory encoding. For instance, temporal inactivation of the nucleus reunions in rats $5 \mathrm{~min}$ after training the passive avoidance task impaired memory retrieval $24 \mathrm{~h}$ later, an effect that disappeared if inactivation was performed 90 or $360 \mathrm{~min}$ post training (Davoodi et al. 2011).

To probe the role of the midline thalamus in systems consolidation, we reanalyzed a unique data set from a prospective four-session fMRI study, which contrasted recent and progressively more remote memory retrieval over the course of $90 \mathrm{~d}$ (Takashima et al. 2006). In this paradigm, subjects studied 320 landscape photographs on day 1 (remote set). After a short rest/ sleep period on day 1 , and again on days 2,30 , and 90 , subjects studied new sets of 80 photographs each (recent sets), followed by scanning sessions in which subjects performed a recognition memory test on 240 randomly intermixed photographs (remote, recent, new) with a confidence rating. Takashima et al. (2006) showed that retrieval-related hippocampal activity decreased, whereas mPFC activity increased over time. Here we extended these findings by performing functional connectivity analyses with a focus on the midline thalamus.

We hypothesized mid-thalamic mediation between the mPFC, hippocampus, and representational areas during the retrieval of memories that have not been fully consolidated yet. Since we had landscape photographs as stimuli, we expected the

(C) 2015 Thielen et al. This article is distributed exclusively by Cold Spring Harbor Laboratory Press for the first 12 months after the full-issue publication date (see http://learnmem.cshlp.org/site/misc/terms.xhtml). After 12 months, it is available under a Creative Commons License (AttributionNonCommercial 4.0 International), as described at http://creativecommons. org/licenses/by-nc/4.0/. 

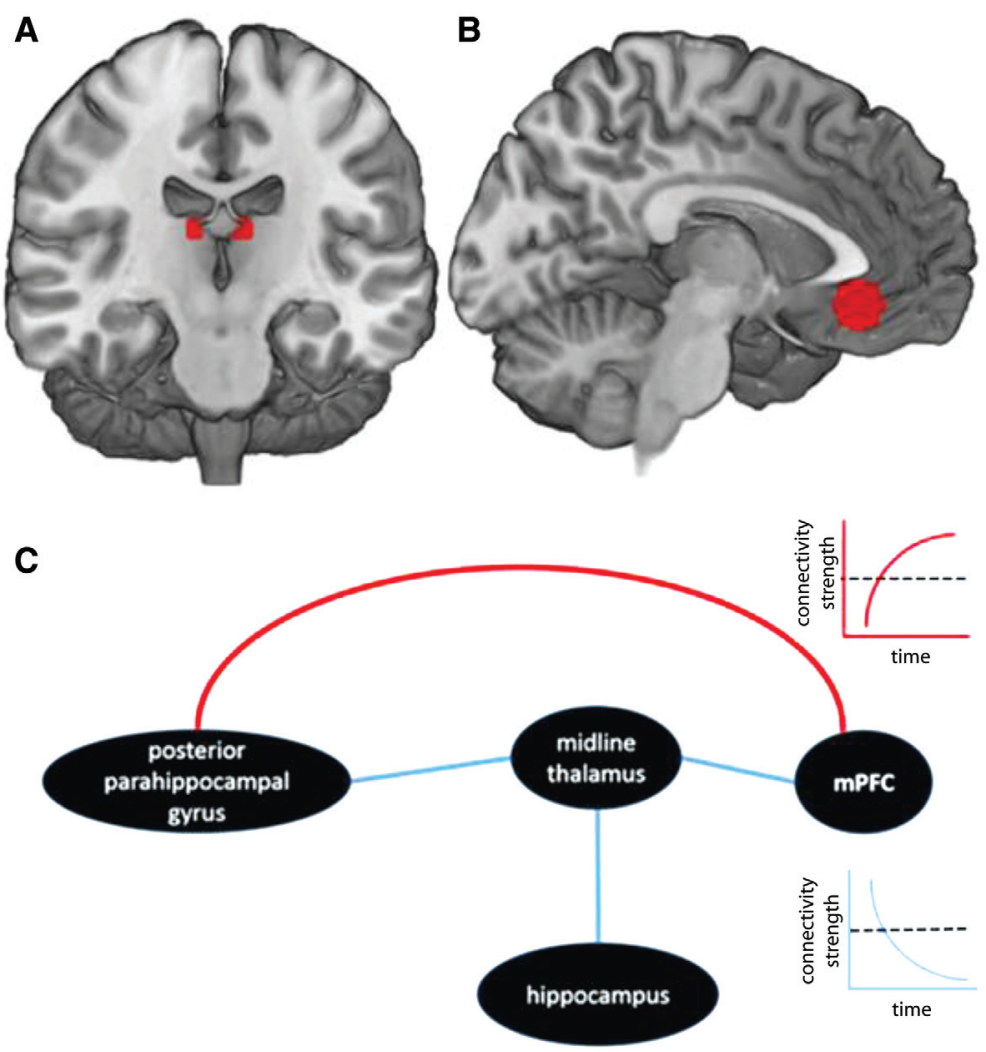

Figure 1. In $(A)$ the midline thalamus and in $(B)$ the mPFC seeds used in the functional connectivity analyses are depicted. The thalamic seed was defined by WFU_pickatlas (midline nucleus) implementing the dilate function (1.3D), but excluding all voxels outside the thalamus. We note that because dilating and smoothing cause an "extention," so that the thalamic seed region is not restricted to the true midline nucleus. Nearby thalamic nuclei, such as the anterior and medial dorsal nuclei, may contribute to the results we report. The mPFC seed was defined as an 8-mm sphere centered around the peak of the remote memory effect (peak MNI coordinate $[-2,32,-10]$ ) initially reported by Takashima et al. 2006. In C, a schematic depiction of the hypothesis is depicted. Blue lines indicate midline thalamus functional connectivity that are increased on day 1 relative to a baseline (for the retrieval of 2-h old memories) and then decreased over the time course of $90 \mathrm{~d}$ following a power law function. The red line indicates the increasing connectivity of the MPFC to the same posterior parahippocampal cortex region that decreased its functional connectivity with the midline thalamus over the time course of $90 \mathrm{~d}$. Black dotted lines are suggestive for the baseline functional connectivity of the recent condition.

parahippocampal cortex (Epstein 2005) to serve as representational area. We assumed that thalamic mediation during retrieval is increased at the early stage of consolidation, but diminishes over time while the interaction between $\mathrm{MPFC}$ and representational areas increases.

Subject demographics and details of the behavioral task, data acquisition, and preprocessing can be found in Takashima et al. (2006). To test our new hypotheses, we performed two psychophysiological interaction (PPI) analyses, one with a midline thalamic seed and another with an mPFC seed. PPI is a method of estimating the functional connectivity, or coupling, between a brain region (seed) and the rest of the brain with relation to a psychological measure (experimental conditions), with the assumption that if two areas coactivate during a specific process, they are functionally connected. In detail, we modeled the trials in which the participants correctly recognized the picture as studied with high confidence, and contrasted those trials that were in the initial study session on day 1 (remote hits) to those in the four recent study sessions (recent hits). For each seed region and session, the necessary PPI parameters (physiological factor, psychological factor, and interaction factor) were calculated prior to the GLM (see O'Reilly et al. 2012). For the physiological factor the first eigenvariate of the time course of the voxels within the seed region was extracted. Next, the psychological factor was created. Here, the onset times for remote hits and recent hits were temporally convolved with the canonical hemodynamic response function (weighted with +1 for remote and -1 for recent hit condition). Finally, an interaction factor was calculated as an interaction term of physiological and psychological factors. Then, for every scan session (day 1-day 90) a separate GLM was conducted with the PPI regressors (physiological, psychological, and interaction factors) of a seed region together with the other experimental condition regressors: onsets of remote hits, recent hits, new [correct rejections], null [fixation cross], misses [incorrect responses] temporally convolved with the hemodynamic response function provided by SPM8, and the six motion regressors derived from realignment during preprocessing. A high-pass filter was implemented using a cut-off period of 128 sec. Then, the subject-specific contrast images for the interaction term of each scan session were used as inputs for the second-level random effects analysis. Analogous to Takashima et al. (2006) we used power laws in the second-level analysis to probe changing functional connectivity over the four scan sessions, weighting the four regressors (day 1day 90) with the following: decrease in connectivity with time $3.0,-0.5,-1.0$, -1.5 and increase $-3.0,0.5,1.0,1.5$.

Statistically, all analyses were initially thresholded at $P=0.001$ (voxel level, uncorrected) and only cluster-size surviving family-wise error (FWE) correction for multiple comparisons were considered significant. Given our a priori hypothesis, we additionally reduced our search space by applying small-volume correction within the hippocampus and the mPFC. We defined spheres with a 10-mm diameter centered at the maxima of the recent and remote memory effects in the original report (left hippocampus: $\mathrm{MNI}=-32,-10,-18$, right hippocampus: $\mathrm{MNI}=32,-4,-26$, and mPFC: $\mathrm{MNI}=-2,32,-10$; Takashima et al. 2006).

As reported previously, recognition memory performance for recent items remained above chance level $\left(d^{\prime}>1.7\right)$ and stable over time $\left(F_{(3,19)}=0.64\right.$, nsec). Remote items were also remembered above chance level $\left(d^{\prime}>0.45\right)$, but performance deteriorated over time $\left(F_{(3,19)}=28.1, P<0.001\right)$.

Using a thalamic midline seed for our first PPI analysis (Fig. 1 ), we found, in line with our hypothesis, increased functional connectivity with the mPFC, hippocampus, posterior parahippocampal gyrus, and BA6 during successful retrieval of items learned prior to rest/sleep as compared with retrieval of items that were studied just prior to scanning on day 1 that declined over the course of $90 \mathrm{~d}$ (Fig. 2). As for the BA6, while this area is commonly associated with motor tasks, Tanaka et al. (2005) showed its involvement in updating spatial and verbal information suggesting a memory updating role of this structure while retrieving 


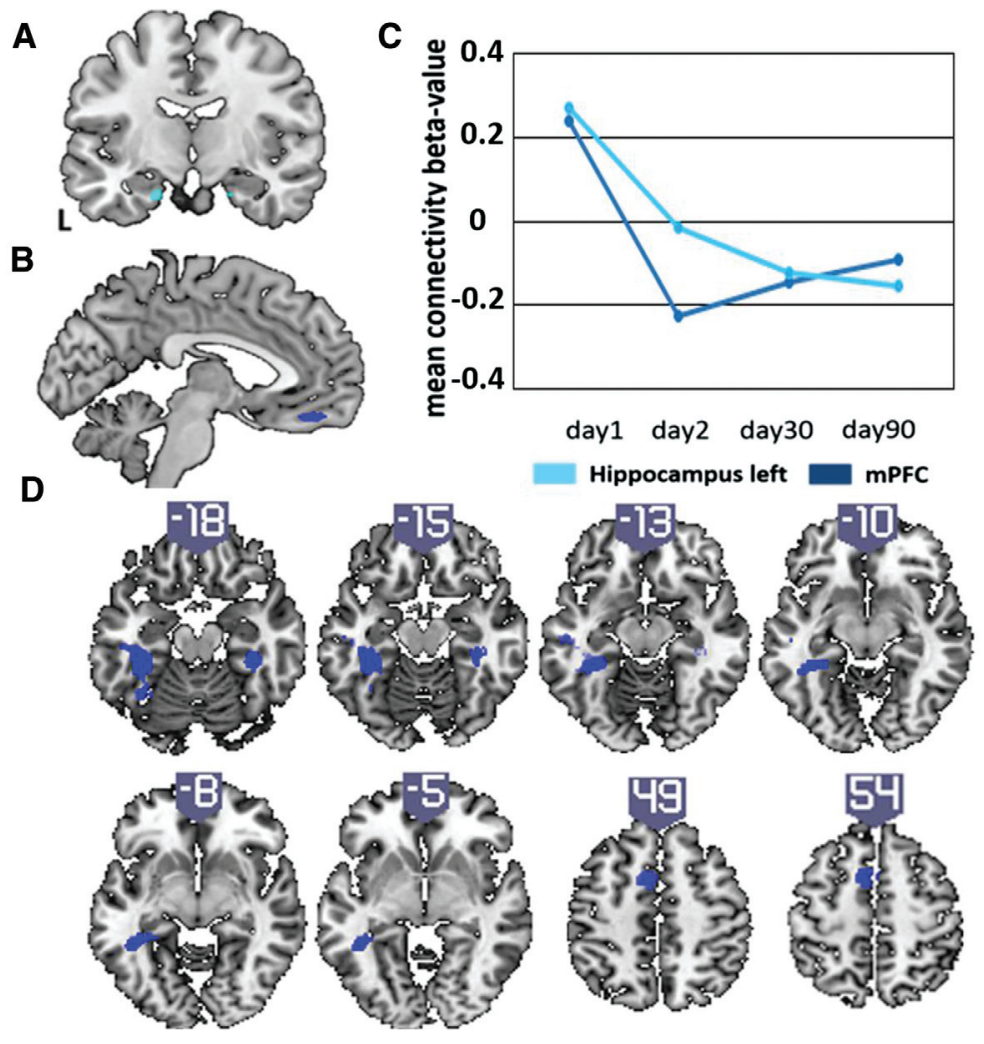

Figure 2. Midline thalamus functional connectivity decrease over time. Midline thalamic functional connectivity maps are overlaid onto a standard brain (MNI). (A) Clusters in the left hippocampus (cyan; maxima at $\mathrm{MNI}=-24,-10,-24$, FWE corrected, $P<0.05$ ) and $(B)$ the $\mathrm{mPFC}$ (dark blue; maxima at $\mathrm{MNI}=-2,38,-16$, FWE corrected, $P<0.04$ ) decreased their functional connectivity with the midline thalamus over time. The right hippocampal cluster just missed the statistical significance $(P=0.092)$. (C) The mean connectivity $\beta$ values of the left hippocampus (cyan) and the mPFC (dark blue) clusters are plotted over the time course of $90 \mathrm{~d}$. (D) The left and right posterior parahippocampal gyri (dark blue; left peak $\mathrm{MNI}=-32,-40,-14$; FWE corrected, $P<0.01$; right peak $\mathrm{MNI}=38,-40,-26$; FWE corrected, $P<0.02$ ), and Brodmann area 6 in the frontal lobe (dark blue; $\mathrm{MNI}=-8,6,52$; FWE corrected, $P<0.03$ ) showed decreased functional connectivity over time in the whole-brain analysis.

memories at an early stage of consolidation. This may indeed be taking place in our study.

Using an mPFC seed in our second analysis, we probed mPFC-posterior parahippocampal gyrus connectivity by selecting the parahippocampal cluster, defined by decreasing connectivity with the thalamic seed, as reduced search space. Here we found progressively increasing mPFC connectivity with an area largely overlapping with the thalamic connectivity findings (Fig. 3).

Taken together, we observed that the retrieval of 2-h old memories that might have undergone initial consolidation is accompanied by a transient thalamic connectivity increase to the hippocampus, mPFC and posterior parahippocampal cortex. This connectivity increase weakened over the course of 90 d. Importantly, the mPFC connectivity with an overlapping posterior parahippocampal region that showed a strong connectivity with the midline thalamus in the first analysis, increased its strength with time. This pattern of results may indicate that during the short rest/sleep period on day 1 , offline consolidation process had caused initial steps of consolidation. Retrieval of such "partly" consolidated memories may require more hippocampalmPFC cooperation, as compared with memories that are still fully hippocampally dependent or already neocortically represented. With time the role of midline thalamus and hippocampus appear to diminish such that connectivity between the MPFC and a specific representational area is associated with successful retrieval.

In what follows, we will discuss our findings in light of two different, but not mutually exclusive, models. It is important to note that the functional connectivity parameters are only indicative of functional interactions between brain regions in a given task, which provide only correlational evidence without direction or causality. Moreover, PPI parameters do not provide information about the underlying neuronal mechanisms and cannot reveal whether different brain regions share the computation of information or if one brain region provides information for another. Nonetheless, our findings are consistent with models that are based on neurophysiological data describing functionally specific thalamo-cortical interactions (Slotnick et al. 2002) that enable feature binding (Engel et al. 1997). In this framework, the retrieval of "partly" consolidated memories may require increased midline thalamic support in binding or integrating cortically distributed information. With time, the synaptic strength between the different cortical areas (here the $\mathrm{mPFC}$ and posterior parahippocampal region) increases, rendering midline thalamic binding processes less relevant.

An alternative interpretation is that the midline thalamus is involved in adjusting the activity level of cortical structures relevant for mnemonic processes. As described by Van der Werf et al. (2003) and Pereira de Vasconcelos and Cassel (2015) the midline thalamic nuclei (and intralaminar nuclei) may control the dispersion of activity providing the appropriate cortical activation level necessary to carry out a cognitive process. This assumption is in line with the findings of Xu and Südhof (2013), which provide evidence in mice that the mPFC controls hippocampal activation levels during memory encoding via the nucleus reuniens. They showed also that this interaction determines how specific/generalized a memory trace becomes. The authors proposed that increased hippocampal activation allowed less prominent memory features to be incorporated into the overall memory of an event, rendering these memories as more specific. Whereas the hippocampus keeps specific representations of individual memory episodes, the cortex abstracts common features from multiple memories (Xu and Südhof 2013). This means that through systems consolidation, memories may become generalized. Based on this model, one may speculate that the midline thalamic functional connectivity parameter reflects an increased need for mediation that may "compensate" for the loss of specificity due to initial systems consolidation. In other words, to retrieve "partly" consolidated memories, the mPFC may utilize the midline thalamus to increase the activity of hippocampal and posterior representation neurons to reinstate the specific memory trace. When the memories have become fully consolidated, the "linking nodes" in the hippocampus are weakened, rendering $\mathrm{MPFC}$ mediation of hippocampal activation via midline 


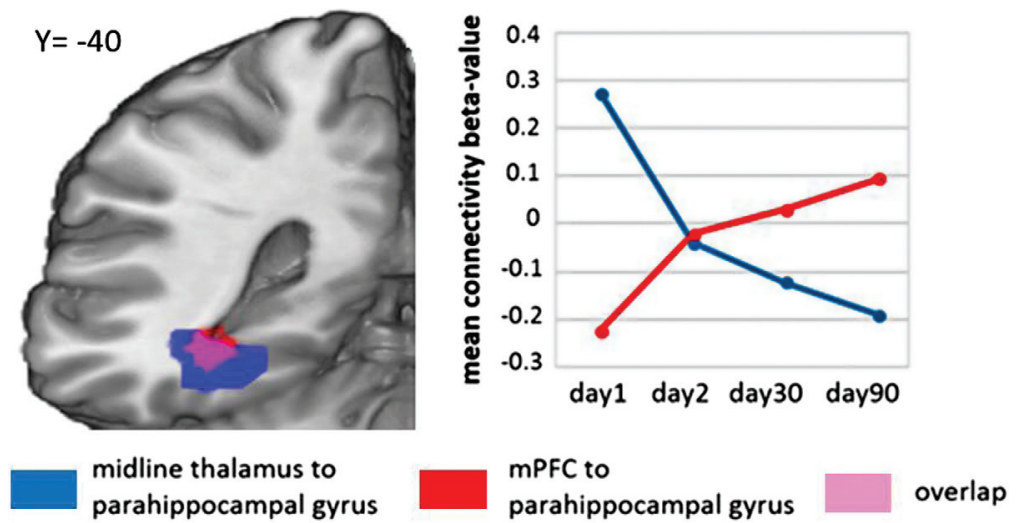

Figure 3. $\mathrm{mPFC}$ functional connectivity change over time. Midline thalamic and mPFC functional connectivity maps are overlaid onto a standard brain (MNI). Increasing functional connectivity with mPFC and decreasing functional connectivity with midline thalamus overlapped in the left posterior parahippocampal gyrus. (Red) cluster that increased functional connectivity with mPFC over $90 \mathrm{~d}$ (maxima at $\mathrm{MNI}=-34,-40,-8$; FWE corrected, $P<0.05$ ). (Blue) cluster that decreased functional connectivity with midline thalamus over $90 \mathrm{~d}$. (Pink) overlap between the two regions (left panel). Mean connectivity $\beta$ values of the mPFC (red) and midline thalamus (blue) cluster over the time course of $90 \mathrm{~d}$ (right panel).

thalamus irrelevant. Although manipulations by Xu and Südhof targeted memory encoding, the role of the midline thalamus during the early stage of consolidation has also been described: Davoodi et al. (2011) showed that midline nuclei are involved in retrieval of memories that are in an early stage of consolidation. They found that inactivation of midline nuclei shortly before a probe trail, at a 24-h delay, disrupts memory performance. Moreover, evidence for an early and transient role of the midline nuclei has also been provided by Davoodi et al. (2011). Here, the authors found that reversible inactivation of midline nuclei disrupted memory performance in a paired-associate task only if the injections were given after a 5-min delay but not at 90- or 360-min delays post training. However, our findings are not entirely in line with findings in rodents. For instance, Loureiro et al. (2012), which used the Morris water-maze task, found increased C-Fos expression in the midline thalamus after a probe trial with a long delay ( $25 \mathrm{~d}$ ) compared with a probe trial with a short delay ( $5 \mathrm{~d}$ ). Moreover, lesioning this region had no effect on acquisition or retrieval after a short delay, but it had an effect on memory retrieval after a long delay. These findings appear to show that the midline nuclei are not involved in the retrieval of memories that are in an "early stage" but in a "later stage" of consolidation. One way to reconcile this discrepancy is that mental schema could play a role in acceleration of consolidation. Memory can benefit from existing schema, and in case newly encoded information can fit into existing knowledge, consolidation is speeded up (Tse et al. 2007). Having instructed subjects to relate the picture to their knowledge (e.g., "Where do you think the picture is?"), might have activated their prior knowledge readily incorporating new information into their existing schema, leading to accelerated consolidation process. However, this speculation needs to be tested further across different species and tasks.

Since our seed region could not be restricted exactly to the midline thalamus, the dynamic changes we observed for midline thalamic connectivity may also be governed by other thalamic nuclei, such as the medial dorsal or anterior thalamus. Van der Werf et al. (2003) proposed that midline and medial dorsal/anterior thalamus subserve two related functions in declarative memory. Whereas the midline thalamus supplies the necessary cortical activation needed to carry out a cognitive process, the medial dor$\mathrm{sal} /$ anterior thalamic nuclei are more involved in focusing on the material that has to be remembered. Thus, our findings may reflect aspects of both processes: (a) integration (binding) of distributed memory items (model one above) and (b) supporting necessary cortical activation (model two above). In other words, the dynamic changes in functional connectivity we report here may be the product of related processes carried out by different thalamic nuclei that are anatomically close to each other and not distinguishable by fMRI as used here. Therefore, the assumed underlying mechanisms that change over the time course of systems consolidation, as described in model one or model two, are not mutually exclusive.

To summarize, the midline thalamic nuclei appear to be involved in memory retrieval at an early stage of memory consolidation before the retrieval network becomes mainly based on neocortical connectivity between representational areas and the $\mathrm{mPFC}$. It seems that midline nuclei are most important when initial consolidation processes have taken place but their involvement decreases continuously after $24 \mathrm{~h}$. During this transient phase, they appear to interact closely with the hippocampus, $\mathrm{mPFC}$ and parahippocampal representation areas. Our results suggest an extension of classical models of systems consolidation by showing a transient role of thalamic midline structures during the reorganization of memories over time. We extended the findings of Takashima et al. (2006) that showed a shift of activity from the hippocampus to the mPFC by demonstrating an associated change in functional connectivity. Our results are in line with a model in which thalamic midline nuclei serve as hub between the hippocampus, mPFC and a representational area in the posterior parahippocampal cortex during memory retrieval at an early $(2 \mathrm{~h})$ stage of consolidation.

\section{Competing interest statement}

The authors declare no conflict of interests.

\section{Acknowledgments}

J.-W.T. was supported by the ICEMED grant to I. Tendolkar from the Helmholtz Alliance, Germany. G.F. is supported by a European Research Council advanced investigator grant (NEUROSCHEMA, ERC R0001075).

\section{References}

Aggleton JP, Brown MW. 1999. Episodic memory, amnesia, and the hippocampal-anterior thalamic axis. Behav Brain Sci 22: $425-489$.

Aggleton JP, Desimone R, Mishkin M. 1986. The origin, course, and termination of the hippocampothalamic projections in the macaque. $J$ Comp Neurol 243: 409-421.

Aggleton JP, Dumont JR, Warburton EC. 2011. Unraveling the contributions of the diencephalon to recognition memory: a review. Learn Mem 18: 384-400.

Alvarez P, Squire LR. 1994. Memory consolidation and the medial temporal lobe: a simple network model. Proc Natl Acad Sci 91: $7041-7045$.

Amaral DG, Cowan WM. 1980. Subcortical afferents to the hippocampal formation in the monkey. J Comp Neurol 189: 573-591. 
Bontempi B, Laurent-Demir C, Destrade C, Jaffard R. 1999. Time dependent reorganization of brain circuitry underlying long-term memory storage. Nature 400: 671-675.

Cassel JC, Pereira de Vasconcelos A, Loureiro M, Cholvin T, Dalrymple-Alford JC, Vertes RP. 2013. The reuniens and rhomboid nuclei: neuroanatomy, electrophysiological characteristics and behavioral implications. Prog Neurobiol 111: 34-52.

Davoodi FG, Motamedi F, Akbari E, Ghanbarian E, Jila B. 2011. Effect of reversible inactivation of reuniens nucleus on memory processing in passive avoidance task. Behav Brain Res 221: 1-6.

DeVito JL. 1980. Subcortical projections to the hippocampal formation in squirrel monkey (Saimira sciureus). Brain Res Bull 5: 285-289.

Engel AK, Roelfsema PR, Fries P, Brecht M, Singer W. 1997. Role of the temporal domain for response selection and perceptual binding. Cereb Cortex 7: 571-582.

Epstein RA. 2005. The cortical basis of visual scene processing. Vis $\operatorname{Cogn}$ 12: 954-978.

Frankland PW, Bontempi B. 2005. The organization of recent and remote memories. Nat Rev Neuroscience 6: 119-130.

Hoover WB, Vertes RP. 2012. Collateral projections from reuniens nucleus of thalamus to hippocampus and medial prefrontal cortex in the rat: a single and double retrograde fluorescent labeling study. Brain Struct Funct 217: 191-209.

Hsu DT, Price JL. 2007. Midline and intralaminar thalamic connections with the orbital and medial prefrontal networks in macaque monkeys. $J$ Comp Neurol 504: 89-111.

Loureiro M, Cholvin T, Lopez J, Merienne N, Latreche A, Cosquer B, Geiger K, Kelche K, Cassel JC, Pereira de Vasconcelos A. 2012. The ventral midline thalamus (reuniens and rhomboid nuclei) contributes to the persistence of spatial memory in rats. J Neurosci 32: 9947-9959.

O'Reilly JX, Woolrich MW, Behrens TE, Smith SM, Johansen-Berg H. 2012. Tools of the trade: psychophysiological interactions and functional connectivity. Soc Cogn Affect Neurosci 7: 604-609.

Pereira de Vasconcelos A, Cassel JC. 2015. The nonspecific thalamus: a place in a wedding bed for making memories last? Neurosci Biobehav Rev 54: $175-196$.

Slotnick SD, Moo LR, Kraut MA, Lesser RP, Hart J Jr. 2002. Interactions between thalamic and cortical rhythms during semantic memory recall in human. Proc Natl Acad Sci 99: 6440-6443.
Takashima A, Petersson KM, Rutters F, Tendolkar I, Jensen O, Zwarts MJ, McNaughtoin BL, Fernández G. 2006. Declarative memory consolidation in humans: a prospective functional magnetic resonance imaging study. Proc Natl Acad Sci 103: 756-761.

Takehara-Nishiuchi K, McNaughton BL. 2008. Spontaneous changes of neocortical code for associative memory during consolidation. Science 322: 960-963.

Tanaka S, Honda M, Sadato N. 2005. Modality-specific cognitive function of medial and lateral human Brodmann area 6. J Neurosci 25: 496-501.

Tse D, Langston RF, Kakeyama M, Bethus I, Spooner PA, Wood ER, Witter MP, Morris RG. 2007. Schemas and memory consolidation. Science 316: 76-82.

Van der Werf YD, Witter MP, Groenegen HJ. 2002. The intralaminar and midline nuclei of the thalamus. Anatomical and functional evidence for participation in processes of arousal and awareness. Brain Res Brain Res Rev 39: 107-140.

Van der Werf YD, Jolles J, Witter MP, Uylings HBM. 2003. Contribution of thalamic nuclei to declarative memory functioning. Cortex 39: 1047-1062.

van Kesteren MTR, Fernández G, Norris DG, Hermans EJ. 2010. Persistent schema dependent hippocampal neocortical connectivity during memory encoding and postencoding rest in humans. Proc Natl Acad Sci 107: $7550-7555$.

Vertes RP, Hoover WB, Do Valle AC, Sherman A, RodriguezJJ. 2006. Efferent projections of reuniens and rhomboid nuclei of the thalamus in the rat. J Comp Neurol 499: 768-796.

Vertes RP, Hoover WB, Szigeti-Buck K, Leranth C. 2007. Reuniens nucleus of the midline thalamus: link between the medial prefrontal cortex and the hippocampus. Brain Res Bull 71: 601-609.

Wheeler AL, Teixeira CM, Wang AH, Xiong X, Kovacevic N, Lerch JP, McIntosh AR, Parkinson J, Frankland PW. 2013. Identification of a functional connectome for long-term fear memory in mice. PLoS Comput Biol 9: e1002853.

Xu W, Südhof TC. 2013. A neural circuit for memory specificity and generalization. Science 339: 1290-1295.

Received February 6, 2015; accepted in revised form June 22, 2015. 


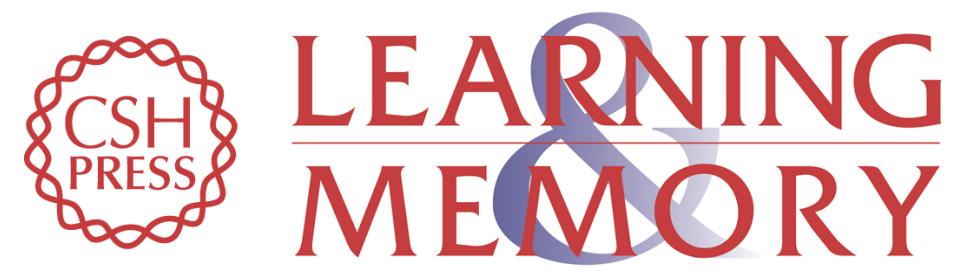

\section{Transient relay function of midline thalamic nuclei during long-term memory consolidation in humans}

Jan-Willem Thielen, Atsuko Takashima, Femke Rutters, et al.

Learn. Mem. 2015, 22:

Access the most recent version at doi:10.1101/Im.038372.115

\begin{aligned} & \hline References $\begin{array}{l}\text { This article cites } 29 \text { articles, } 10 \text { of which can be accessed free at: } \\ \text { http://learnmem.cshlp.org/content/22/10/527.full.html\#ref-list-1 }\end{array} \\ & \begin{array}{r}\text { Creative } \\ \text { Commons } \\ \text { License }\end{array} \begin{array}{l}\text { This article is distributed exclusively by Cold Spring Harbor Laboratory Press for the } \\ \text { first } 12 \text { months after the full-issue publication date (see } \\ \text { http://learnmem.cshlp.org/site/misc/terms.xhtml). After } 12 \text { months, it is available under } \\ \text { a Creative Commons License (Attribution-NonCommercial } 4.0 \text { International), as } \\ \text { described at http://creativecommons.org/licenses/by-nc/4.0/. }\end{array} \\ & \begin{array}{c}\text { Receive free email alerts when new articles cite this article - sign up in the box at the } \\ \text { top right corner of the article or click here. }\end{array} \\ & \begin{array}{l}\text { Service } \\ \text { terting }\end{array}\end{aligned}$

